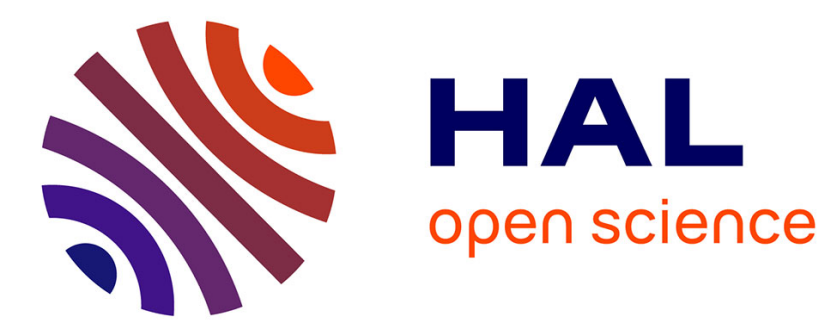

\title{
Biomimetic Autopilot Based on Minimalistic Motion Vision for Navigating along Corridors Comprising U-shaped and S-shaped Turns
}

Julien Serres, Franck Ruffier

\section{- To cite this version:}

Julien Serres, Franck Ruffier. Biomimetic Autopilot Based on Minimalistic Motion Vision for Navigating along Corridors Comprising U-shaped and S-shaped Turns. Journal of Bionic Engineering, 2015, 12 (1), pp.47 - 60. 10.1016/S1672-6529(14)60099-8 . hal-01108274

\section{HAL Id: hal-01108274 \\ https://hal.science/hal-01108274}

Submitted on 22 Jan 2015

HAL is a multi-disciplinary open access archive for the deposit and dissemination of scientific research documents, whether they are published or not. The documents may come from teaching and research institutions in France or abroad, or from public or private research centers.
L'archive ouverte pluridisciplinaire HAL, est destinée au dépôt et à la diffusion de documents scientifiques de niveau recherche, publiés ou non, émanant des établissements d'enseignement et de recherche français ou étrangers, des laboratoires publics ou privés. 
Cite this article:

Serres J. and Ruffier F. (2015)

Biomimetic autopilot based on minimalistic motion vision for navigating along corridors comprising $U$-shaped and S-shaped turns

Journal of Bionic Engineering, vol. 12, No. 1, pp. 47-60 .

\title{
Biomimetic autopilot based on minimalistic motion vision for navigating along corridors comprising U-shaped and S-shaped turns
}

\author{
Julien R. Serres and Franck Ruffier \\ Aix-Marseille Université, CNRS, ISM UMR 7287, Biorobotics Lab, 13288, Marseille Cedex 09, \\ France \\ email: \{julien.serres; franck.ruffier\}@univ-amu.fr
}

Corresponding author: Julien Serres, email : julien.serres@univ-amu.fr

Keywords: optic flow; collision avoidance; body saccades; insect flight; biorobotics; biomimetics.

Running title: Biomimetic autopilot based on minimalistic motion vision 


\begin{abstract}
.
A bio-inspired autopilot is presented, in which body saccadic and intersaccadic systems are combined. This autopilot enables a simulated hovercraft to travel along corridors comprising L-junctions, U-shaped and Sshaped turns, relying on minimalistic motion vision cues alone without measuring its speed or distance from walls, in much the same way as flies and bees manage their flight in similar situations. The saccadic system responsible for avoiding frontal collisions triggers yaw body saccades with appropriately quantified angles based simply on a few local optic flow measurements, giving the angle of incidence with respect to a frontal wall. The simulated robot negotiates stiff bends by triggering body saccades to realign its trajectory, thus traveling parallel with the wall along a corridor comprising sharp turns. Direct comparison shows that the performance of this new body saccade-based autopilot closely resembles the behavior of a fly using similar body saccade strategy when flying along a corridor with an S-shaped turn, despite the huge differences in terms of the inertia.
\end{abstract}

\title{
1. Introduction
}

Searching for inspiration from flying insects is an attractive approach because these creatures' flying skills have been perfected during several hundred million years of evolution. Flying insects have been found to rely mainly on the optic flow (OF) [1,2], which can be defined as the ratio between their relative speed and their distance from obstacles in the surrounding environment [3]. Specialized neurons called lobula plate tangential cells (LPTCs) seem to serve as wide-field OF sensors in head stabilization and visual guidance processes (Flies: [4,5]; Honeybees: [6]; see review [7]). In addition, the trajectories of flies [8-11] and bees [12] have been found to usually consist of straight flight sequences interspersed with rapid turns termed saccades. Intersaccadic sequences, in which flying insects move in the purely translation mode, enable the LPTCs to assess the purely translational OF, which depends on several parameters including their distance from objects. Some biomimetic experiments were performed to better understand how the intersaccadic interval (ISI) contributes to flying insects' behavior, assuming that intersaccadic system results in a constant forward speed $[13,14]$.

Few studies have been performed using evolutionary approaches (se review [15]) in which it has been attempted to use only a few pixels to generate obstacle avoidance behavior based on visual motion cues, however the OF was not eventually used after (evolving for) 20 generations [16]. Simulations have been performed using a minimalistic OF approach to 3-D obstacle avoidance problems, taking very little into account the dynamic and closed-loop aspects [17].

It was recently suggested using a biorobotic approach that honeybees' wall-following behavior may involve the use of a dual OF regulator combined with a heading lock system [18], which makes a robot follow lateral walls and keep its speed proportional to the width of the corridor (Eq. 15 in [19]). This lateral OF regulation principle may explain how flying insects are able to control their speed depending on the narrowness of the environment [20-23] while avoiding walls [1,24] when travelling along straight and tapered corridors, keeping a straight course.

However, flying insects are perfectly able to fly along a straight corridor without their body being perfectly aligned with the corridor axis [24,25]: honeybees can collect a reward at the end of straight and apparent Lshaped corridors by adjusting their course appropriately [26], and flies can avoid frontal obstacles encountered in a circular arena [8,9] and travel along a corridor with an S-shaped turn [27]. These ethological findings cannot be explained solely in terms of a dual OF regulator coupled to a heading lock system maintaining a straight course $[18]$. 
The so-called 'OF balance' hypothesis initially put forward to explain bees' centering behavior [1] has been implemented in a large number of visually guided wheeled robots [28-31] travelling along various corridors, including even some with bends [29,30] and L-junctions [30,31], but none of these robots were able to cope with U-turns or S-shaped turns, as far as we know. Other collision-avoidance algorithms based on the OF have been implemented onboard microflyers [32-34] using a series of open-loop commands to trigger saccades to avoid frontal obstacles. Most of these previous robotic solutions required thousands of pixels. In other studies, the robots had only one or two degrees of freedom (DOFs) [13,28-34] or they were endowed with OF-based guidance systems based on very slow OFs of less than $70 \%$ s $[31,33,34]$.

In our quest to explain how free-flying insects behavior in complex corridors, we equipped our Simulated Fully Actuated Hovercraft (SFAH) with a bio-inspired autopilot controlling each of its DOFs on the basis of the OF at levels of $90 \%$ and even more. The novel collision-avoidance algorithm developed for this purpose estimates the angle of incidence $\hat{\imath}$ between the robot and a frontal obstacle using just two local OF measurements, which are made in the frontal part of the field of view (FOV). An additional parameter, the OF divergence [35], is used to trigger yaw saccades of an appropriate amplitude to prevent any crash. The strategy presented here differs from those developed elsewhere in several respects:

- the robot's speed is variable: our SFAH moves at variable forward and side speeds during the ISI, as found to occur in free-flying insects [10,20-23], and concomitantly decelerates and drifts sideway during the body saccades [10,27].

- the amplitude of the body saccades is variable: it can be estimated quantitatively and adjusted continuously (from $-90^{\circ}$ to $+90^{\circ}$ ), as occurs in many free-flying insects $[8,9,11,12,27]$ : this is done here by computing just a few local OF values in order to make the robot's trajectory run parallel to the wall, thus enabling it to negotiate a corridor comprising sharp turns. The present strategy therefore differs radically from those presented in previous studies featuring constant pre-specified saccades [33], saccade durations based on a Gaussian distribution [14] or saccades which were modulated qualitatively using the OF to avoid obstacles efficiently $[13,32,34]$.

Our present simulation makes a robot capable of negotiating sharp turns by endowing it successively with:

- $\quad$ an intersaccadic system based on a dual OF regulator controlling the robot's speed while keeping a constant course between two successive saccades by means of a heading-lock system [18],

- a saccadic system responding to any frontal obstacles detected by triggering body saccades and updating the heading set-point by visually estimating the angle of incidence $\hat{\imath}$ between the robot and the frontal obstacle.

In section 2, the simulation set-up and the SFAH equipped to perform 8 local OF measurements are described in detail. In section 3, our bio-inspired autopilot is presented in detail. In section 4, we describe the trajectories of a SFAH equipped with our bio-inspired autopilot, which were simulated in challenging corridors comprising Ljunctions, U-turns, and S-shaped turns. In section 5, the potential advantages of our bio-inspired autopilot as a means of achieving autonomous robotic navigation are discussed, along with new biological hypotheses. 


\section{Simulation methods}

All the computer-simulated experiments were carried out on a standard computer running the MATLAB/Simulink@ software program at a sampling frequency of $2 \mathrm{kHz}$. This robotic simulation was based on a realistic dynamic model of the hovercraft (about the surge, sway, and yaw axes) (Fig. 1a) including the Coriolis effects.

Neither the texture on the walls nor the phototransduction processes involved were taken into account in the simulations in order to save computational resources because bees' behaviors (centering response [1] and bees' speed control [20]) were not texture- or contrast-dependent. In addition, the authors of electrophysiological studies on honeybees' motion sensitive neurons, which have been called velocity-tuned neurons (VT neurons), have established that their responses were closely correlated with the motion of the patterns [6]. Models for visual motion detection showing texture- and contrast- independence can be found in [36-38] which differ from the Hassenstein-Reichardt model (HR correlator) originally developed in 1956 [39].

Optoelectronic Local Motion Sensors (LMSs) measuring the OF were simulated here using a black-box model including their optical and temporal characteristics [37,38]: the inter-photoreceptor angle $\Delta \varphi, \Delta t$ range, $\Delta t$ quantification and the effects of noise on the OF measurements .

\subsection{Simulated 2-D environment}

The 2-D environment was simulated by taking a 6000x6000 binary matrix coding with a spatial resolution of $1 \mathrm{~mm}$. The SFAH can therefore navigate in a simulated environment $6 \times 6 \mathrm{~m}$ in size. The binary value ' 1 ' simulates the walls of the environment. This environment matrix was used to find the distance $D_{\varphi}$ from the local OF measurement pointing at the azimuthal angle $\varphi$ to the nearest obstacle (Fig. 1b).

\subsection{Physical model of the hovercraft mimicking flying insects' locomotor mode}

Throughout this study, the wind will be not considered. Our hovercraft (Fig. 1a) is fully actuated by four thrusters driving it the 3 DOFs on the horizontal plane: (i) the two rear thrusters drive the hovercraft along the surge axis, (ii) a pair of lateral thrusters drives the robot along the sway axis, and (iii) the two rear thrusters that are also controlled differentially, drive the robot around the $\psi$-axis (see [18] for details). A SFAH (Fig. 1b) is an attractive vehicle because the forward speed $V_{f}$ and the side speed $V_{s}$ are uncoupled in the 2-D horizontal plane, in line with what occurs in bees along the surge axis [40] and the sway axis [41]. The equations of motion, including the Coriolis effects, can be written as follows in the local frame of the hovercraft:

$$
\begin{aligned}
& m \cdot \dot{V}_{f}-2 m \cdot V_{s} \cdot \dot{\psi}+\zeta_{f} \cdot V_{f}=2 K_{T} \cdot U_{b a t t} \cdot u_{f} \\
& m \cdot \dot{V}_{s}+2 m \cdot V_{f} \cdot \dot{\psi}+\zeta_{s} \cdot V_{s}=K_{T} \cdot U_{b a t t} \cdot u_{s} \\
& \quad \dot{d} \\
& J \cdot \frac{\dot{\psi}}{d t}+\mu \cdot \dot{\psi}=2 K_{T} \cdot U_{b a t t} \cdot r \cdot u_{\psi}
\end{aligned}
$$

These equations include 8 parameters: the mass $m(857 \mathrm{~g})$, the moment of inertia $J\left(0.0125 \mathrm{~kg} \cdot \mathrm{m}^{2}\right)$, the moment arm $r(0.095 \mathrm{~m})$, the linear surge $\zeta_{f}$ and sway $\zeta_{s}$, and rotational $\mu$ viscous friction coefficients, whose values depend on the lift fan control signal (tables I and II) assuming that $\zeta_{f}=\zeta_{s}$ and noted $\zeta$ in table I, the input voltage 
$U_{\text {batt }}(8.2 \mathrm{~V})$, and $K_{T}(0.094 \mathrm{~N} / \mathrm{V})$, the static gain relating the thruster's force to the voltage applied. All the transfer functions used in the simulations are described in table I, which corresponds to figure 4 in the study by [18].

\subsection{Optic flow generated by the hovercraft's own motion}

The hovercraft travels over a flat-floored corridor with bends. The local OF generated throughout the azimuthal angle $\varphi$ (denoted $\omega_{\varphi}$ ) was computed as follows, based on the geometry of the corridor, the position of the SFAH and its motion [42]:

$\omega_{\varphi}=-\dot{\psi}+\frac{1}{D_{\varphi}}\left(V_{f} \cdot \sin \varphi-V_{s} \cdot \cos \varphi\right)$

where $D_{\varphi}$ is the distance from the wall at the azimuthal angle $\varphi, V_{f}$ and $V_{s}$ are the forward and side speeds, respectively, and $\dot{\Psi}$ is the robot's yaw velocity (Fig. 1b).

The hovercraft perceives both the translational and rotational OFs (Eq. 2). However, any yaw disturbance will introduce a rotational OF component $(-\dot{\Psi}$ in Eq. 2$)$, which will be quickly compensated for by a custommade heading-lock system controlling the rear thrusters differentially in the closed-loop mode (Fig. 4A in [18]). The yaw rate feedback loop adjusts the robot's yaw rate so as to keep the yaw rotational velocity as low as possible via a micro-gyrometer, which makes the robot experience purely translational OF [18].

\subsection{Local motion sensors on the simulated hovercraft}

The Local Motion Sensors (LMSs) were simulated in the present study using theoretical local OF measurements, to which we added a Gaussian noise and a temporal resolution observed in the robotic model. The Gaussian noise is defined by a normal distribution $N(\mu=0, \sigma=0.55 \mathrm{~ms})$. A temporal resolution of $0.5 \mathrm{~ms}$ is applied on the theoretical time lag $\Delta t$ in order to generate jitter in the $\mathrm{OF}$ measurements at high $\mathrm{OF}$, as found to occur in physical robots' OF sensors [38]. The simulated LMS refreshes the OF measurements at a sampling rate of $12 \mathrm{~Hz}$ : this average refresh rate was assessed during LMS characterization under good indoor lighting conditions [43]. A sine-law gradient (Eq. 3, already used by [44] was introduced into the inter-receptor angle $\Delta \phi_{\varphi}$ of each of the 8 LMSs (hence, 8 local OF measurements) in our minimalistic visual system (Fig. 1c) to mimic insects' compound eye [45].

$$
\Delta \phi_{\varphi}=\Delta \phi_{90^{\circ}} \cdot \sin \varphi
$$

The inter-receptor angles $\Delta \phi_{\varphi}$ were therefore smaller in the frontal part of the visual system than in the lateral part (Table III) as observed in the honeybee's compound eye [45]. 


\section{Bio-inspired autopilot}

\subsection{The intersaccadic system: forward and side control systems while keeping a constant heading}

A dual OF regulator controls the surge and sway axes of our SFAH [18]. It requires only two feedback signals (denoted $\omega_{\text {Rmeas }}$ and $\omega_{\text {Lmeas }}$ ) originating from the two sides of the FOV, and each OF regulator has its own OF set-point:

(i) the first lateral OF regulator is a unilateral OF regulator that adjusts the hovercraft's lateral thrust so as to keep the higher of the two lateral OFs perceived equal to the sideways OF set-point (noted $\omega_{\text {setside }}$ ). The outcome is that the distance to the nearest wall becomes proportional to the hovercraft's forward speed $V_{f}$, as determined in (ii).

(ii) the second lateral OF regulator is a bilateral OF regulator, which adjusts the hovercraft's forward thrust so as to keep the sum of the two OF measurements (right and left) equal to a forward OF set-point (denoted $\omega_{\text {setFwd }}$ ). As the result of these two intertwined feedback loops, the forward speed:

- tends to be proportional to the local corridor width in straight or diverging corridors because the OF measurements originate mainly from facets oriented at azimuthal angles of $\pm 90^{\circ}$,

- or tends to be adjusted in converging corridors with respect to frontolateral obstacles because the highest OF measurements originate mainly from facets oriented at azimuthal angles of $\pm 30^{\circ}$.

The present SFAH was equipped with a set of 8 facets oriented at azimuthal angles of $\pm 90^{\circ}, \pm 45^{\circ}, \pm 30^{\circ}$, and $\pm 15^{\circ}$ (Fig. 1c). Facets at angles of $\pm 90^{\circ}, \pm 45^{\circ}$, and $\pm 30^{\circ}$ were used to generate the two feedback signals feeding the dual OF regulator. The lateral FOV was restricted to azimuthal angles of $\pm 30^{\circ}$ because it was established that the OF measured in regions facing towards the frontolateral part of the FOV have a strong impact on the flight speed $[21,23,27]$. The visual feedback signals $\omega_{\text {Lmeas }}$ or $\omega_{\text {Rmeas }}$ were computed using an frontolateral OF criterion originating from eyes facing at angles of $\pm 30^{\circ}, \pm 45^{\circ}$ and $\pm 90^{\circ}$ in line with Eq. 5, i.e., by taking the maximum value of the OF measurements weighted with a set of static gains $K_{\varphi}$ computed as follows:

$$
\begin{aligned}
& K_{\varphi}=\frac{1}{\sin ^{2}(\varphi)} \\
& \omega_{L_{\text {meas }}}=\max \left(K_{ \pm 30^{\circ}} \cdot \omega_{ \pm 30^{\circ}} ; K_{ \pm 45^{\circ}} \cdot \omega_{ \pm 45^{\circ}} ; K_{ \pm 90^{\circ}} \cdot \omega_{ \pm 90^{\circ}}\right) \\
& =\max \left(4 \cdot \omega_{ \pm 30^{\circ}} ; 2 \cdot \omega_{ \pm 45^{\circ}} ; \omega_{ \pm 90^{\circ}}\right)
\end{aligned}
$$

The forward $\left(\omega_{\text {setFwd }}=130 \%\right.$ s $)$ and sideways $\left(\omega_{\text {setSide }}=90 \%\right.$ s $)$ OF set-points were chosen with a view to reaching a safe forward speed $V_{f \infty}=0.48 \mathrm{~m} / \mathrm{s}(\mathrm{Eq} .6)$ and a safe distance from the nearest lateral wall $D_{ \pm 90^{\circ} \infty}=0.31 \mathrm{~m}(\mathrm{Eq}$. 7) in the steady state, with a given corridor width of $D=1 \mathrm{~m}$.

$$
\begin{aligned}
& V_{f \infty}=\frac{\omega_{\text {SetSide }} \cdot\left(\omega_{\text {SetFwd }}-\omega_{\text {SetSide }}\right)}{\omega_{\text {SetFwd }}} \cdot D \\
& D_{ \pm 90^{\circ}}=\frac{\omega_{\text {SetFwd }}-\omega_{\text {SetSide }}}{\omega_{\text {SetFwd }}} \cdot D
\end{aligned}
$$




\subsection{Saccadic system: yaw control system}

\subsubsection{Detection of frontal obstacles triggering body saccades}

To make the robot capable of avoiding a forthcoming frontal collision, the FOV was divided into its frontolateral and frontal parts. The two facets oriented at azimuthal angles of $\pm 15^{\circ}$ were allocated to the frontal part of the FOV. The OF measured with the $\pm 15^{\circ}$ facets increases monotonically with the distance from a frontal obstacle, thus making it possible to detect imminent frontal collisions (see the 'saccade initiation' block in Fig. 2a) and to trigger a body saccade with a higher level of priority than the intersaccadic system.

If the robot's side speed $V_{s}$ and yaw velocity $\dot{\Psi}$ are both null, the robot's speed will be aligned with its own body axis. It can therefore be proved that the sum of the two symmetrical OFs with respect to the body axis (Eq. 8) can be defined as an index to the OF divergence and is therefore inversely proportional to the Time-ToContact (TTC) (see Eq. 9).

$\omega_{\varphi}+\omega_{-\varphi}=\frac{V_{f}}{D_{0^{\circ}}} \cdot \sin (2 \varphi)$

where $\omega_{\varphi}$ is the OF generated throughout the azimuthal angle $\varphi, V_{f}$ is the robot's forward speed and $D_{0^{\circ}}$ is the distance to the obstacle at $\varphi=0^{\circ}$. The TTC can therefore be calculated as follows:

$\frac{1}{T T C}=\frac{V_{f}}{D_{0^{\circ}}}=\frac{\omega_{\varphi}+\omega_{-\varphi}}{\sin (2 \varphi)}$

The sum of the OF generated throughout the $\pm 15^{\circ}$ azimuthal angles can therefore be used to trigger a body saccade at an angle determined by the OF outputs when the sum $\omega_{15^{\circ}}+\omega_{-15^{\circ}}$ is greater than a given threshold value. With $\varphi=15^{\circ}$ :

$\omega_{15^{\circ}}+\omega_{-15^{\circ}}=\frac{1}{2 \cdot T T C}$

In all our simulations, the threshold value was fixed at $35 \%$, corresponding to a $T T C=0.82 \mathrm{~s}$ (e.g., a robot moving at a $V_{f}=0.61 \mathrm{~m} / \mathrm{s}$ towards an obstacle $1 \mathrm{~m}$ ahead). The $T T C$ threshold value depends directly on the robot's yaw time constant $\tau_{\psi}$. Assuming $\tau_{\psi} \approx 72 \mathrm{~ms}$ (under $u_{\text {LiffFan }}=36 \%$, see table II), since this time constant is more than 11 times smaller than the TTC value, the robot will be able to reach its new steady state heading without colliding with the frontal obstacles encountered. The main advantage of using the OF divergence to detect imminent collision is that this criterion (Eq. 10) does not depend on the geometry of the corridor or the robot's orientation with respect to the corridor.

\subsubsection{Estimation of the angle of incidence used to compute the new heading angle}

An OF-based algorithm was developed for estimating the angle of incidence $i$ (Fig. 1d) between the robot's longitudinal axis and a frontal obstacle (see the 'angle of incidence estimation' block in Fig. 2a). It can be proved from Fig. 1d that the distance $D_{ \pm \varphi}$ from an obstacle at an azimuthal angle $\pm \varphi$ is:

$D_{ \pm \varphi}=D_{0^{\circ}} \cdot \frac{1+\tan ^{2} \varphi}{1 \pm \tan \varphi \cdot \tan i} \cdot \cos \varphi$ 
Assuming that both the robot's side speed $V_{s}$ and its yaw velocity $\dot{\Psi}$ are null, the robot's speed is aligned with its own body axis. It can therefore be proved that:

$\omega_{\varphi}-\omega_{-\varphi}=\left(\frac{V_{f}}{D_{\varphi}}-\frac{V_{f}}{D_{-\varphi}}\right) \cdot \sin \varphi=-2 \cdot \sin ^{2} \varphi \cdot \frac{V_{f}}{D_{0^{\circ}}} \cdot \tan i$

and then from equations (8) and (12), we obtain:

$\frac{\omega_{\varphi}-\omega_{-\varphi}}{\omega_{\varphi}+\omega_{-\varphi}}=-\tan \varphi \cdot \tan i$

We can now calculate the angle of incidence $i$ as follows:

$i=-\tan ^{-1}\left(\frac{1}{\tan \varphi} \cdot \frac{\omega_{\varphi}-\omega_{-\varphi}}{\omega_{\varphi}+\omega_{-\varphi}}\right)$ with $\left.\varphi \in\right] 0 ; 90^{\circ}[$

An angle of $\pm 90^{\circ}$ is then added to the angle of incidence angle $i$ in order to determine the next heading set-point $\psi_{\text {set-point }}$ (see the 'updating the heading set-point' block in Fig. 2a) depending on the obstacle avoidance side (left or right), which depends directly on the sign of $\omega_{45^{\circ}}^{m}-\omega_{-45^{\circ}}^{m}$ (see the 'side of the saccade' block in Fig. 2a). The updated heading set-point $\Psi_{\text {set-point }}$ causes the robot to travel forward in parallel with a surface after its body saccade (Fig. 2a).

The method used here to estimate the angle of incidence (Eq. 14) requires only two symmetrical local OF measurements around the focus of expansion. In this study, the angle of incidence $i$ is estimated only with the robot's facets oriented at an angle of $\pm 30^{\circ}$ (see Eq. 15 and the 'angle of incidence estimation' block in Fig. 2a). This gives a better estimate of the incidence angle $\hat{i}$ than that obtained when the facets are oriented at an angle of $\pm 15^{\circ}$, which is very sensitive to noise and to small measurement errors. Facets oriented at $\pm 45^{\circ}$ are too laterally positioned to be able to look simultaneously at a single frontal obstacle. Our choice of $\pm 30^{\circ}$ between the facets therefore constitutes the best trade-off between $\pm 15^{\circ}$ and $\pm 45^{\circ}$ as values of the angle of incidence $\hat{i}$.

$\hat{i}=-\tan ^{-1}\left(\sqrt{3} \cdot \frac{\omega_{30^{\circ}}^{m}-\omega_{-30^{\circ}}^{m}}{\omega_{30^{\circ}}^{m}+\omega_{-30^{\circ}}^{m}}\right)$

Lastly, a minimum $I S I=2.25 \mathrm{~s}$ prevents any new body saccades from being triggered immediately after the previous one (Fig. 2a). The dynamic constraint about the minimum ISI value depends on both the TTC and the response time of the heading-lock system in the closed loop mode $(\mathrm{Tr})$ as follows:

- $\quad I S I \geq T T C: T T C$ value is set at $0.82 \mathrm{~s}$ as explained in $\$ 3.2 .1$ presenting the detection of frontal obstacles,

- $\quad I S I \geq T r$ : the minimum $I S I$ value required to be higher than the response time of the heading-lock system in the closed loop mode $(T r=2.2 \mathrm{~s}$ as in Fig. $3 \mathrm{~g})$.

However, as the body saccade is defined in terms of angles, the inhibition time of the intersaccadic system (Fig. 2b) was not constant in our simulations because it depends on the yaw dynamics (Table II). The body saccades were therefore generated in the closed loop mode. An overview of the full control scheme can be seen in figure $2 \mathrm{~b}$ showing the inputs and the outputs of each subsystem controlling each of three DOFs of the robot. 


\section{Results of the simulations}

The corridor width used here was similar to that used in ethological experiments on bees. The SFAH was tested in corridors ranging from $0.8 \mathrm{~m}$ to $1.2 \mathrm{~m}$ in width as described in $\S 4.1$ and $\S 4.2$, and then in a tapered corridor ranging from $0.8 \mathrm{~m}$ to $2.6 \mathrm{~m}$ in width as described in $\S 4.3$, and lastly in a challenging corridor including a $0.8 \mathrm{~m}$ wide S-shaped turn, as described in $\$ 4.4$.

\subsection{Corridor-following in the presence of a $45^{\circ}$ bend}

The full control scheme (Fig. 2b) was first tested onboard our SFAH travelling along a 1-meter wide corridor in which a $45^{\circ}$ bend was located $2.5 \mathrm{~m}$ from the starting-point (Fig. 3a). Starting at various initial positions, our SFAH was found to be able to follow an angular corridor (Fig. 3a). Some of the parameters of our SFAH (Fig. $3 b)$ travelling along this corridor are presented in figures $3 \mathrm{c}-\mathrm{g}$. During the first 2.2 seconds of the simulation, the robot aligns its headingwith the corridor axis before following the right wall between $\mathrm{t}=2.2 \mathrm{~s}$ and $\mathrm{t}=6.6 \mathrm{~s}$, as dictated by the intersaccadic system. The SFAH then reaches the final operating point $\left(V_{f \infty}=0.48 \mathrm{~m} / \mathrm{s}, D_{-90^{\circ} \infty}=\right.$ $0.31 \mathrm{~m}$ ) as predicted by equations 6 and 7 (Figs. $3 \mathrm{~cd}$ ).

At $\mathrm{t}=6.6 \mathrm{~s}$, the sum of the OF measurements performed while facing at an angle of $\pm 15^{\circ}$ reaches the $\mathrm{OF}$ threshold value (Fig. 3f): the saccade generator turns off the intersaccadic system (Fig. 2b) and jointly triggers a body saccade turn by updating the heading set-point $\Psi_{\text {set-point }}$ computed by the saccade generator (Fig. 2a), and therefore, by adding an angle of $29^{\circ}$ to the previous heading set-point $\Psi_{\text {set-point }}=90^{\circ}$ (Fig. 3e). The angle of incidence $\hat{\imath}$ (Eq. 15) is not correctly estimated here by the visual system because the two local OF measurements oriented at an angle of $\pm 30^{\circ}$ are not obtained while facing the same frontal wall (the front one is at an angle of $+30^{\circ}$, and the right one, at an angle of $-30^{\circ}$ ) at the moment when the angle $\hat{\imath}$ is estimated (Eq. 15), which generates a small bias of $16^{\circ}$ (Fig. $3 \mathrm{e}$ ). At t $=9.2 \mathrm{~s}$, the robot reaches its new heading set-point $\Psi_{\text {set-point }}=119^{\circ}$ (Fig. 3e) and the intersaccadic system is again turned on, but at $\mathrm{t}=10 \mathrm{~s}$, since the robot is too near the right wall, a second saccade with an amplitude of $30^{\circ}$ (Fig. 3f) is triggered (Fig. 3e). At $t=12.6 \mathrm{~s}$, the robot reaches its final heading set-point $\Psi_{\text {set-point }}=149^{\circ}$ (Fig. 3e), the intersaccadic system is again turned on and the robot adopts wallfollowing behavior (Fig. 3b). It can again be observed that the robot is able to follow the left wall, even if it was not travelling perfectly in parallel with the wall (Fig. 3b).

\subsection{Wall-following behavior in a maze containing several L-junctions}

The full control scheme (Fig. 2b) was also tested onboard our SFAH in a corridor with a mean length of $17 \mathrm{~m}$ and a variable width $(D=0.8 \mathrm{~m}$ in Fig. $4 \mathrm{a} ; D=1.2 \mathrm{~m}$ in Fig. $4 \mathrm{~b} ; D=1 \mathrm{~m}$ in Fig. $4 \mathrm{c})$ containing four L-junctions. Starting at various initial positions, our SFAH was able to travel along a maze (Fig. 4a-c). Both the robot's speed and the lateral position are controlled by the dual OF regulator, which ensures that the robot's speed and the distance from the nearest obstacle are constantly proportional to the local corridor width, as predicted by the simulated dual OF regulator (see §3.1). The mean speed $V=0.29 \pm 0.02 \mathrm{~m} / \mathrm{s}$ in the case of a local corridor width of $D=0.8 \mathrm{~m}$, as shown in Fig. $4 \mathrm{a}$, while $V=0.34 \pm 0.02 \mathrm{~m} / \mathrm{s}$ in the case of $D=1 \mathrm{~m}$, as shown in Fig. $4 \mathrm{~b}$, and $V$ $=0.39 \pm 0.03 \mathrm{~m} / \mathrm{s}$ in the case of $D=1.2 \mathrm{~m}$; as shown in Fig. $4 \mathrm{c}$ : our SFAH is therefore able to adjust its speed in proportion to the width of the corridor, as found to occur in flying insects [20-23,27]. 
The blue trajectory shown in figure $4 \mathrm{~d}$ was selected from the set of 7 trajectories presented in figure $4 \mathrm{c}$. The hovercraft images superimposed on this trajectory give the SFAH's position and its orientation at every 2.8s. It can be seen from figure $4 \mathrm{~d}$ that our SFAH succeeded in swiftly negotiating a challenging 1-meter wide corridor including no less than four L-junctions via either their salient angle $\left(90^{\circ}\right)$ or their returning angle $\left(270^{\circ}\right)$. It can be seen from this trajectory (Fig. 4d) that the SFAH's speed was relatively constant along the straight sections of the corridor, while its heading was updated at each L-junction.

Performances of our intersaccadic system in terms of the speed control

The mean speed is $V=0.35 \pm 0.21 \mathrm{~m} / \mathrm{s}$ along the trajectory shown in figure $4 \mathrm{~d}$. However, if we consider the first straight section of the corridor in figure $4 \mathrm{~d}$, we obtain $V=0.47 \pm 0.07 \mathrm{~m} / \mathrm{s}$ at $2.2 \mathrm{~s} \leq t \leq 10 \mathrm{~s}$, which is similar to the values predicted by equation 6. Our intersaccadic system therefore ensures that a speed is maintained constant in the straight section, whereas the speed and side control signals are clamped to zero in the angular section, forcing the speed to decrease when the absolute value of the orientation error is upper than $2^{\circ}$ (Fig. 2b).

\section{Performances of our intersaccadic system in terms of the distance control}

The mean distance is $D_{90^{\circ}}=1.01 \pm 1.15 \mathrm{~m}$ and $D_{-90^{\circ}}=0.76 \pm 1.02 \mathrm{~m}$ along the trajectory shown in figure $4 \mathrm{~d}$, whereas the minimum distance is $\min \left\{D_{90^{\circ}}(\mathrm{t}) ; D_{-90^{\circ}}(\mathrm{t})\right\}=0.32 \pm 0.09 \mathrm{~m}$ along this same trajectory: the fact that this minimum distance is close to those predicted by equation 7 shows that our intersaccadic system is able to maintain a constant distance from the nearest lateral wall at a given corridor width.

\subsection{Following a corridor with a tapering angle of $27^{\circ}$ and $a 3^{\circ}$ bend}

In the third simulated experiment, the bio-inspired autopilot was tested (Fig. 2b) onboard our SFAH in a converging/diverging corridor (corresponding to a tapering angle of $27^{\circ}$ ) with a 0.80 -meter wide straight section (oriented at an angle of $63^{\circ}$ ) located midway. Starting at various initial positions, our SFAH proved to be able to navigate successfully along a challenging corridor (Fig. 5a). Figure 5b shows a SFAH's trajectory selected from the set of 8 trajectories shown in figure 5a. The forward speed profile in figure 5c shows that the robot's speed is either adjusted to ensure environmental clearance or held constant at a straight junction, or even slowed down drastically during saccades. Since the frontolateral $\pm 30^{\circ}$ facets detect the lateral OF before the lateral $\pm 90^{\circ}$ facets, the robot can be said to be endowed with a kind of prediction horizon [18] that helps it to reject lateral disturbances in a highly converging section, as observed to occur in insects flying along corridors with a variable width $[1,21,23,27]$.

\subsection{Travelling through an $S$-shaped turn}

In the fourth simulated experiment, the full control scheme (Fig. 2b) was tested onboard our SFAH in a corridor comprising a $0.8 \mathrm{~m}$-wide S-shaped turn. Starting at various initial positions and orientations, our SFAH proved to be able to travel successfully along an S-shaped corridor (Fig. 6a). Body saccades occurred along the whole Sshaped turn (Fig. 6b), as also reported by [27] in the case of blowflies. A typical robot trajectory (Fig. 6c) was compared with a typical blowfly trajectory (Fig. 6d) in a similar corridor configuration; similar behavior was observed in both cases, despite the huge difference in term of the inertia. Most of the simulated trajectories involved four saccades (Fig. 6e) of various amplitudes ranging between $30^{\circ}$ and $90^{\circ}$ (Fig. 6f), which is reminiscent of the findings obtained on blowflies [27]. Lastly, we compared the changes with time in the body 
yaw angle (in the case of the SFAH in Fig. 6g and that of the blowfly in Fig. 6h) in an S-shaped corridor, in which 5 yaw body-saccades were required to overcome the S-shaped turn. In figures $6 \mathrm{~g}$-h, the body orientation profile was similar in both cases: our biorobotic approach therefore yielded a better understanding of this aspect of insects' flight. 


\section{Discussion}

\subsection{What is not insect-like and what is insect-like in our model}

The present model is not insect-like in the following respects:

- The model is limited to the horizontal plane. Insects fly in 3 dimensions.

- The model only includes 16 photoreceptors, 8 local OF measurements and 2 fused wide-field OF measurements. Insects may be endowed with more than one thousand pixels performing local OF measurements, as well as dozens of wide-field neurons sensitive to the OF.

- The model does not include any artificial spiking neurons. Insects are equipped with both depolarizing (analog processing) neurons and spiking neurons.

- $\quad$ The SFAH's inertia is 10,000 times greater than that of flies.

The present model is insect-like in the following respects:

- Like insects, the model obeys a holonomic locomotor mode in the horizontal plane, where the three DOFs (forward, sideward and yaw) are uncoupled [40,41].

- $\quad$ The model has an insect-like minimalistic compound eye which is sensitive to the OF [44].

- The gradient of the eye's inter-ommatidial angle is included in the model of the OF measurement [45].

- The minimalistic compound eye can be subdivided into frontolateral and frontal regions [21].

- All the sensors used in the model have biological counterparts in insects: the OF is sensed by the compound eye $[4-6,37,46]$, the mechanical rotational speed is sensed by the halteres, the heading angle with respect to a global frame is sensed by the dorsal rim or the ocelli $[1,7]$.

- $\quad$ Like insects, the model requires no groundspeed or distance measurements.

- The model accounts for insects' ability to cope with various tunnel widths and configurations [20$24,26,27]$.

\subsection{Robotic contribution}

The novel OF-based autopilot presented here enables a SFAH to accomplish collision-avoidance manoeuvers in complex corridors comprising U-turns and S-shaped turn. The present bio-inspired autopilot uses the $\mathrm{OF}$ and a heading-lock system to perform corridor-following tasks without having to use any conventional metric sensors such as active distance sensors or speed sensors. Frontolateral obstacles are detected by means of a minimalistic visual system involved in an intersaccadic system based on a dual OF regulator responsible for the robot's translations $(\$ 3.1)$, and frontal obstacles are detected by means of two local OF measurements oriented at an azimuthal angle of $\pm 15^{\circ}$ involved in a saccadic system (\$3.2). Simulation results prove the efficiency of this saccadic system, in which the robot's angle of incidence (Eq. 15) with respect to the frontal obstacle is used to turn of the correct angle to make the robot follow the next wall.

This bio-inspired autopilot including both intersaccadic and saccadic systems is the first step toward designing a deft, lightweight, power-lean visuomotor control system that could be implemented on novel free-flying insectlike tiny robots [47] by extending the FOV to the ventral and dorsal parts [23,34]. In addition, the present simulated visual processing system meet the low requirements of the recent curved artificial compound eyes called CurvACE [48]. For this purpose, CurvACE would require an inter-receptor sinus gradient (Eq. 3).

\subsection{Biological contribution}


This is the first visuomotor control scheme including both intersaccadic and saccadic systems (Fig. 2) which possibly accounts for insects' flight behavior in complex corridors comprising apparent L-junctions [26], or S-shaped turn [27]. The robot's maximum rotational velocity (in this case, 50\%) is therefore much lower than that of flying insects (up to $3000 \%$ s in $[11,27]$ ), that is why our SFAH requires a large ISI lasting several seconds (as compared with about 100ms in the blowfly [27]) to force the robot to reduce its speed down to almost zero in order to avoid a frontal obstacle. However, our SFAH does not decelerate before performing a body saccade but only during the saccade, as blowflies have been found to do [10,27], and a sideways drift can occur due to Coriolis effects, as occurs in blowflies [10], which is not compensated for in our model (Fig. 2b) during rotations but only during translations, as suggested by previous authors in their model for a blowfly (called the "CyberFly" [13]). Our biological hypothesis was tested onboard a SFAH mimicking the locomotion mode of honeybees, whose surge and sway dynamics are uncoupled on both the surge axis [40] and the sway axis [41]. Besides, our simulated visual system was equipped with 8 local OF measurements set at similar inter-receptor angles to those detected in the honeybee's compound eye [45], and the OF measurement range was also in the range of that observed in the VT neurons studied in bees [6]. Our biorobotic approach therefore helps to understand the flight strategy at work in blowflies as well as how HSE-cells might be involved in controlling horizontal turns. The HSE-cells [4] are sensitive to horizontal movements and show maximum sensitivity at $\varphi=$ $\pm 15^{\circ}$ [46], corresponding to the orientations in which the present autopilot measures the OF in order to compute the incidence angle $\hat{\imath}$ (Eq. 15).

The present SFAH is equipped with a heading-lock system which keeps its heading constant between two saccades; in flying insects, a similar system based on polarized light cues may exist $[1,7]$.

\section{Conclusion}

The novel bio-inspired autopilot for performing corridor-following tasks developed in this study enables a SFAH to travel along complex corridors on the basis of OF cues without ever having to measure the speed or the distance from the walls. In many respects, our SFAH mimics insects' locomotor abilities and the visual processes at work when they are flying on the horizontal plane. By subdividing the FOV into frontolateral and frontal zones, our bio-inspired autopilot equipped with both intersaccadic and saccadic systems makes our SFAH travel forward, sideways, and turn using only a set of 8 local OF measurements. Our present saccadic system differs considerably from the strategies proposed in previous studies in that it triggers body saccades defined in terms of a quantified saccade angle which is estimated on the basis of just a few local OF measurements. The biorobotic approach enables us to "kill two birds with one stone", because it yields robotic solutions which require much fewer pixels than conventional approach, while providing a possible explanation for free-flying insects' ability to travel safely along complex unknown corridors.

\section{Acknowlegements}

We thank the Reviewers for their stimulating and helpful comments, J. Diperi (a mechanical engineer) for his expert technical assistance, F. Roubieu, S. Viollet, N. Franceschini, and T. Raharijaona for fruitful discussions and J. Blanc for revising the English manuscript.

\section{References}

[1] Srinivasan M V. Honeybees as a model for the study of visually guided flight, navigation, and biologically inspired robotics. Physiological Reviews, 2011, 91(2), 413-460. 
[2] Egelhaaf M, Boeddeker N, Kern R, Kurtz R, Lindemann J P. Spatial vision in insects is facilited by shaping the dynamic of visual input through behavioral action. Frontiers in Neural Circuits, 2012, 6.

[3] Gibson J J. The perception of the visual world, Boston, Houghton Mifflin,1950.

[4] Hausen K. The lobula-complex of the fly: structure, function and signification in visual behaviour. Photoreception and vision in invertebrates, M. A. Ali (Ed.), NATO ASI Series, Series A: Life Science, Plenum, 1984, 74, 523-559.

[5] Krapp H G, Hengstenberg R. Estimation of self-motion by optic flow processing in single visual interneurons, Nature, 1996, 384, 463-466.

[6] Ibbotson M R. Evidence for velocity-tuned motion sensitive descending neurons in the honeybee. Proceedings of the Royal Society B, 268(1482), 2195-2201.

[7] Taylor G K, Krapp H G. Sensory systems and flight stability: What do insects measure and why? In: Casas J (Ed.), Advances in insect physiology: insect mechanics and control, 34, 2007, 231-316.

[8] Tammero L F, Dickinson M H. The influence of visual landscape on the free flight behavior of the fruit fly Drosophila melanogaster. Journal of Experimental Biology, 205(3), 2002, 327-343.

[9] Censi A, Straw A D, Sayaman R W, Murray R M, Dickinson M H. Discriminating External and Internal causes for heading changes in freely flying Drosophila. PLOS Computational Biology, 2013, 2(9), e1002891.

[10] Land M F, Collett T S. Chasing behaviour of houseflies (Fannia canicularis). Journal of Comparative Physiology, 1974, 89, 331-357.

[11] Schiltra C, van Hateren J H. Blowfly flight and optic flow. I. Thorax kinematics and flight dynamics. Journal of Experimental Biology, 1999, 202, 1481-1490.

[12] Boeddeker N, Dittmar L, Stüzzl, Egelhaaf M. The fine structure of honeybee head and body yaw movements in a homing task. Proceedings of the Royal Society B, 2012, doi: 10.1098/rspb.2009.2326.

[13] Lindemann J P, Weiss H, Möller R and Egelhaaf M. Saccadic flight strategy facilitates collision avoidance: closed-loop performance of a cyberfly. Biological Cybernetics, 2008, 98, 213-227.

[14] Reiser M L and Dickinson M H. A test bed for insect-inspired robotic control. Philosophical Transactions of The Royal Society Of London A, 2003, 361, 2267-2285.

[15] Floreano D, Husbnads P, Nolfi S. Evolutionary robotics. Springer handbook of robotics, 2008, 1423-1451.

[16] Floreano D, Epars Y, Zufferey J C, Mattiussi C. Evolution of spiking neural circuits in autonomous mobile robots, International Journal of Intelligent Systems, 2006, 21(9), 1005-1024.

[17] Neumann T R, Huber S A, Bülthof H H. Minimalistic approach to 3D obstacle avoidance behavior from simulated evolution, Artificial Neural Networks (ICANN'97), 1997, Springer Berlin Heidelberg, 715-720.

[18] Roubieu F L, Serres J R, Colonnier F, Franceschini N, Viollet S, Ruffier F. A biomimetic vision-based hovercraft accounts for bees' complex behaviour in various corridors. Bioinspiration \& Biomimetics, 2014, 9, 036003 .

[19] Serres J, Dray D, Ruffier F, Franceschini N. A vision-based autopilot for a miniature air vehicle: joint speed control and lateral obstacle avoidance, Autonomous Robot, 25, 2008, 103-122.

[20] Baird E, Srinivasan M V, Zhang S W, Cowling A. Visual control of flight speed in honeybees. Journal of Experimental Biology, 208, 2005, 3895-3905.

[21] Baird E, Kornfeldt T, Dacke M. Minimum viewing angle for visually guided ground speed control in bumblebees. Journal of Experimental Biology, 213(10), 2010, 1625-1632.

[22] Fry S N, Rohrseitz N, Straw A D, Dickinson M H. Visual control of flight speed in Drosophila melanogaster, Journal of Experimental Biology, 212, 2009, 1120-1130.

[23] Portelli G, Roubieu F L, Ruffier F, Franceschini N. Honeybees' speed depends on dorsal as well as lateral, ventral and frontal optic flows, PLOS ONE, 6(5), 2011, e19486.

[24] Serres J, Masson G, Ruffier F, Franceschini N. A bee in the corridor: centering and wall-following, Naturwissenschaften, 95, 2008, 1181-1187.

[25] Collett T S. Some Operating Rules for the Optomotor System of a Hoverfly During Voluntary Fligh. Journal of Comparative Physiology A, 1980, 138, 271-282. 
[26] Evangelista C, Kraft P, Dacke M, Labhart T, Srinivasan M V. Honeybee navigation : critically examining the role of the polarization compass, Philosophical Transactions of the Royal Society B, 2014, 369(1636), 20130037.

[27] Kern R, Boeddeker N, Dittmar L, Egelhaaf M. Blowfly flight characteristics are shaped by environmental features and controlled by optic flow information. Journal of Experimental Biology, 2012, 215(14), 2501-2514.

[28] Coombs D, Roberts K. Bee-bot: using peripheral optical flow to avoid obstacles. Society of Photo-Optical Instrumentation Engineers (SPIE) Conference Series, Boston, USA, September 1992, 714-721.

[29] Santos-Victor J, Sandini G, Curotto F, Garibaldi S. Divergent stereo in autonomous navigation: From bees to robots. International Journal of Computer Vision, 1995, 14(2), 159-177.

[30] Weber K, Venkatesh S, Srinivasan M V. Insect inspired behaviors for the autonomous control of mobile robots. From living eyes to seeing machines, Srinivasan M V and Venkatesh S (Eds.), Oxford University Press, Oxford, 1997,11, 226-248.

[31] Humbert J S, Hyslop A M. Bioinspired visuomotor convergence. IEEE Transactions on Robotics, 2010, 26(1), 121-130.

[32] Green W E, Oh P Y, Barrows G. Flying insect inspired vision for autonomous aerial robot near-Earth environments. Proceeding of the IEEE Conference on Robotics and Automation (ICRA), New Orleans, USA, April 2004, 2347-2352.

[33] Zufferey J-C, D. Floreano D. Fly-inspired visual steering of an ultralight indoor aircraft. IEEE Transactions on Robotics, 2006, 22(1), 137-146.

[34] Beyeler A, Zufferey J-C, Floreano D. 3D vision-based navigation for indoor microflyers. IEEE International Conference on Robotics and Automation (ICRA), Roma, Italy, April 2007, 1336-1341.

[35] Ancona N, Poggio T. Optical flow from 1D correlation: application to a time-to-crash detector. Proceedings of the $4^{\text {th }}$ International Conference on Conputer Vision, 1993, 209-214.

[36] Srinivasan M V, Poteser M, Kral K. Motion detection in insect orientation and orientation. Vision Research, 1999, 39(16), 2749-2766.

[37] Franceschini N, Riehle A, Le Nestour A. Directionally selective motion detection by insects neurons. In D G Stavenga \& R C Hardie (Eds.), Facets of vision, Springer, Berlin, 1989, 360-390.

[38] Ruffier F, Franceschini N. Optic flow regulation: the key to aircraft automatic guidance. Robotics and Autonomous Systems, 2005, 50(4), 177-194.

[39] Hassenstein B, Reichardt W. Systemtheoretische analyse der zeit-, reihenfolgen-und vorzeichenauswertung bei der bewegungsperzeption des rüsselkäfers chlorophanus. Z. Naturforsch, 1956, 11, 513-524.

[40] Esch H, Natchigall W, Kogge S N. Correlations between aerodynamic output, electrical activity in the indirect flight muscles and flight positions of bees flying in a servomechanically controlled flight tunnel. Journal of Comparative Physiology, 1975, 100, 147-159.

[41] Ellington C P. The aerodynamics of hovering insect flight. III. Kinematics. Philosophical Transactions of The Royal Society Of London B, 1984, 305, 41-78.

[42] Koenderink J, Doorn A. Facts on optic flow. Biological Cybernetics, 1987, 56, 247-254.

[43] Expert F, Viollet S, Ruffier F. Outdoor field performances of insect-based visual motion sensors. Journal of Field Robotics, 2011, 28(4), 974-977.

[44] Franceschini N, Pichon J M, Blanes C. From insect vision to robot vision. Philosophical Transactions of The Royal Society Of London B, 1992, 337, 283-294.

[45] Seidl R A. Die sehfelder und ommatidien-divergenzwinkel von arbeiterin, königen und drohne der honigbiene (Apis mellifica), Ph.D. thesis, Darmstadt: Technische Hochschule Darmstadt, NºDO3046, 1982.

[46] Krapp H G, Hengstenberg R, Egelhaaf M. Binocular contributions to optic flow processing in the fly visual system, Journal of Neurophysiology, 2001, 85(2), 724-734.

[47] Ma K Y, Chirarattananon P, Fuller S. B., Wood R J. Controlled flight of a biologically inspired, insect-scale robot. Science, 2013, 340, 603-607.

[48] Floreano D, Pericet-Camara R, Viollet S, Ruffier F, Brückner A, Leitel R, Buss W, Menouni M, Expert F, Juston R, Dobrzynski M K, L'Eplattenier G, Recktenwald F, Mallot H A, Franceschini N. Miniature curved artificial compound eyes. Proceedings of the National Academy of Sciences of the United States of America (PNAS), 2013, doi: 10.1073/pnas.1219068110. 


\section{Tables}

Table I. Transfer functions in figure 4 in the study by Roubieu et al 2014 [18]

\begin{tabular}{|c|c|}
\hline$H_{c}(s)=K_{c}$ & where $K_{c}=9.17 \cdot 10^{-3} \mathrm{~V} /^{\circ}$ \\
\hline$H_{g}(s)=K_{g}$ & where $K_{\mathrm{g}}=5 \cdot 10^{-3} \mathrm{~V} /(\% \mathrm{~s})$ \\
\hline$G_{\psi}(s)=\frac{\dot{\Psi}(s)}{U_{\psi}(s)}=\frac{\frac{2 K_{T} \cdot U_{\text {batt }} \cdot r}{\mu}}{1+\frac{J}{\mu} \cdot s}=\frac{H_{\psi}}{1+\tau_{\psi} \cdot s}$ & See Table II for values \\
\hline$C_{\psi}(s)=K_{\psi}$ & where $K_{\psi}=4$ \\
\hline$C_{\psi} .(s)=K_{1} \cdot \frac{1+\tau_{1} \cdot s}{s}$ & where $K_{l}=30$ and $\tau_{l}=0.33 \mathrm{~s}$ \\
\hline$G_{V f}(s)=\frac{V_{f}(s)}{U_{f}(s)}=\frac{\frac{2 K_{T} \cdot U_{\text {batt }}}{\zeta}}{1+\frac{m}{\zeta} \cdot s}=\frac{K}{1+\tau \cdot s}$ & See Roubieu et al 2014 [18] \\
\hline$C_{V f}(s)=K_{2} \cdot \frac{1+\tau_{2} \cdot s}{s}$ & where $K_{2}=0.14$ and $\tau_{2}=7 \mathrm{~s}$ \\
\hline$G_{V s}(s)=\frac{V_{s}(s)}{U_{s}(s)}=\frac{\frac{K}{2}}{1+\tau \cdot s}$ & See Roubieu et al 2014 [18] \\
\hline$C_{V s}(s)=K_{3} \cdot \frac{1+a \cdot T \cdot s}{1+T \cdot s}$ & $\begin{array}{c}\text { where } K_{3}=0.15, a=6, \\
\text { and } T=0.2 \mathrm{~s}\end{array}$ \\
\hline
\end{tabular}

Table II. Estimated yaw dynamics depending on the lift fan's control signals

\begin{tabular}{c|c|c|c}
\hline $\begin{array}{c}\text { Forward control } \\
\text { signal value } \\
u_{f}\end{array}$ & $\begin{array}{c}\text { Lift fan } \\
\text { control signal } \\
u_{\text {LiffFan }}\end{array}$ & $\begin{array}{c}\text { Yaw } \\
\text { time constant } \\
\tau_{\psi}(\mathrm{ms})\end{array}$ & $\begin{array}{c}\text { Yaw } \\
\text { static gain } \\
H_{\psi}(\mathrm{rad} / \mathrm{s})\end{array}$ \\
\hline$-6 \%$ & $36 \%$ & 71.72 & 0.906 \\
\hline$-5 \%$ & $37 \%$ & 94.37 & 1.193 \\
\hline$-4 \%$ & $38 \%$ & 108.7 & 1.351 \\
\hline$-3 \%$ & $39 \%$ & 142.4 & 1.530 \\
\hline$-2 \%$ & $40 \%$ & 185.2 & 1.898 \\
\hline$-1 \%$ & $41 \%$ & 185.9 & 2.059 \\
\hline$\geq 0$ & $42 \%$ & 188.2 & 2.205 \\
\hline \hline
\end{tabular}

Table III. Inter-receptor angle $\Delta \phi_{\varphi}$ of each facet in the robot's visual system

\begin{tabular}{ccc}
\multicolumn{3}{c}{ in the robot's visual system } \\
\hline \hline $\begin{array}{c}\text { Azimuthal } \\
\text { angle } \varphi\end{array}$ & $\Delta \phi_{\varphi}$ & $\begin{array}{c}\text { Optic flow } \\
\text { range }\end{array}$ \\
\hline $\pm 90^{\circ}$ & $\Delta \phi_{90^{\circ}}=3.50^{\circ}$ & From $25^{\circ} / \mathrm{s}$ to $350^{\circ} / \mathrm{s}$ \\
$\pm 45^{\circ}$ & $\Delta \phi_{45^{\circ}}=2.50^{\circ}$ & From $18^{\circ} / \mathrm{s}$ to $250^{\circ} / \mathrm{s}$ \\
$\pm 30^{\circ}$ & $\Delta \phi_{30^{\circ}}=1.75^{\circ}$ & From $13 \%$ to $175 \% \mathrm{~s}$ \\
$\pm 15^{\circ}$ & $\Delta \phi_{15^{\circ}}=0.91^{\circ}$ & From $7 \% \mathrm{~s}$ to $91 \% \mathrm{~s}$ \\
\hline \hline
\end{tabular}




\section{Figures}
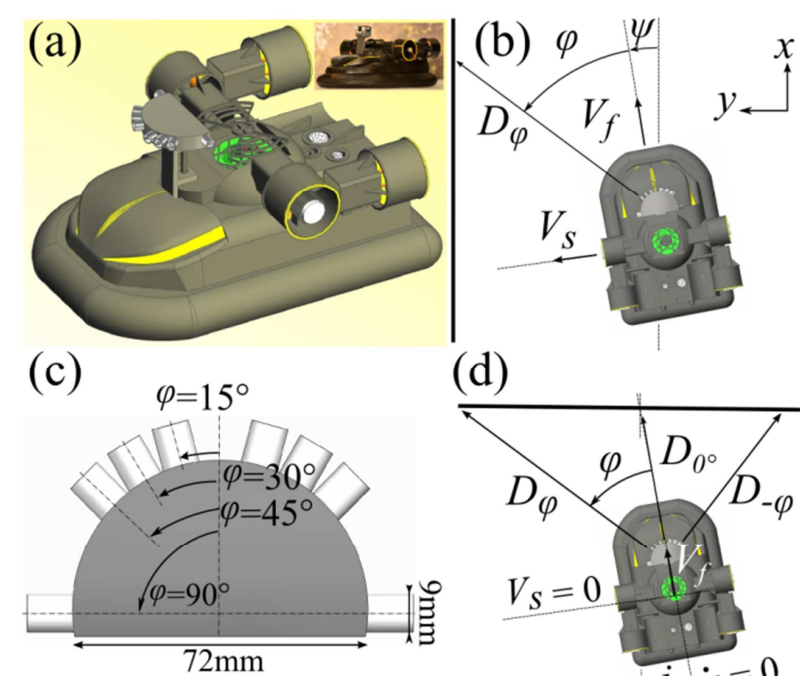

(d)

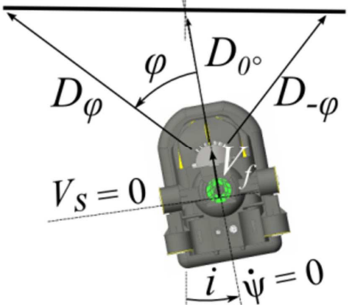

Fig. 1. (a) CAD of the simulated robot (inset: the robot [18]: $0.36 \times 0.21 \times 0.14 \mathrm{~m}$ ). (b) Robot's parameters in a corridor. (c) CAD of the robot's primitive compound eye composed of 8 facets placed on each side of the eye and oriented in four different directions ranging from $15^{\circ}$ to $90^{\circ}$. (d) Robot frontally approaching a wall with an angle of incidence $i$. 
(a)

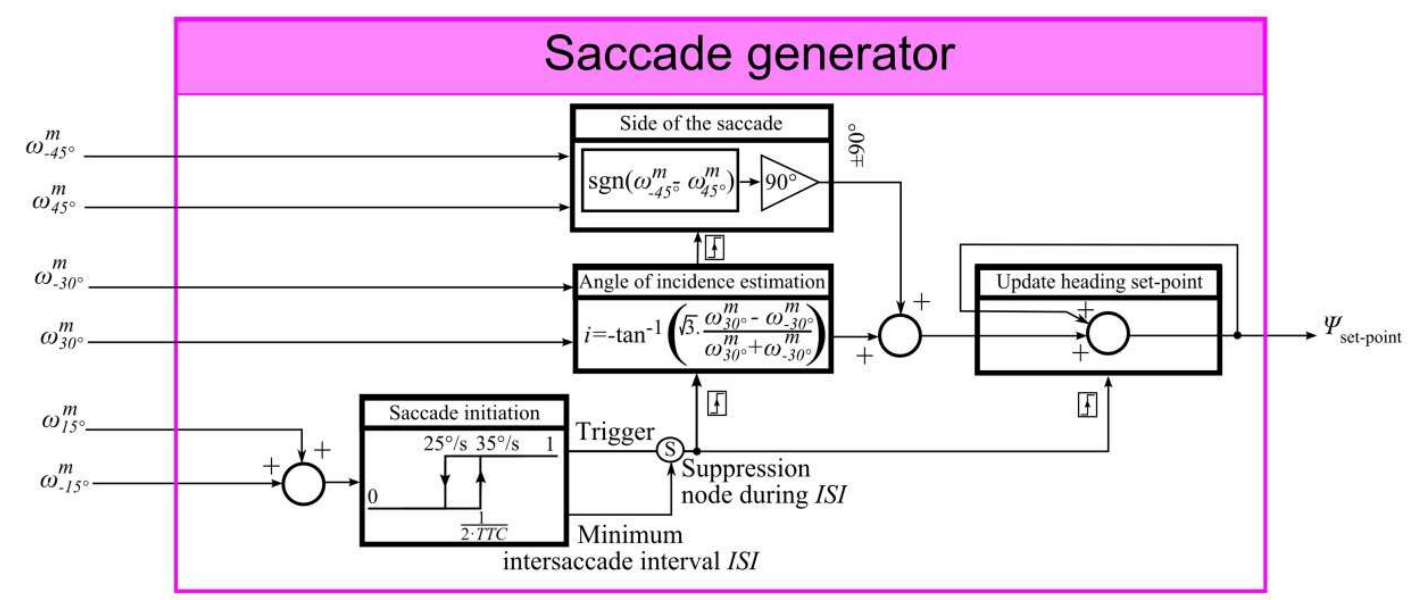

(b)

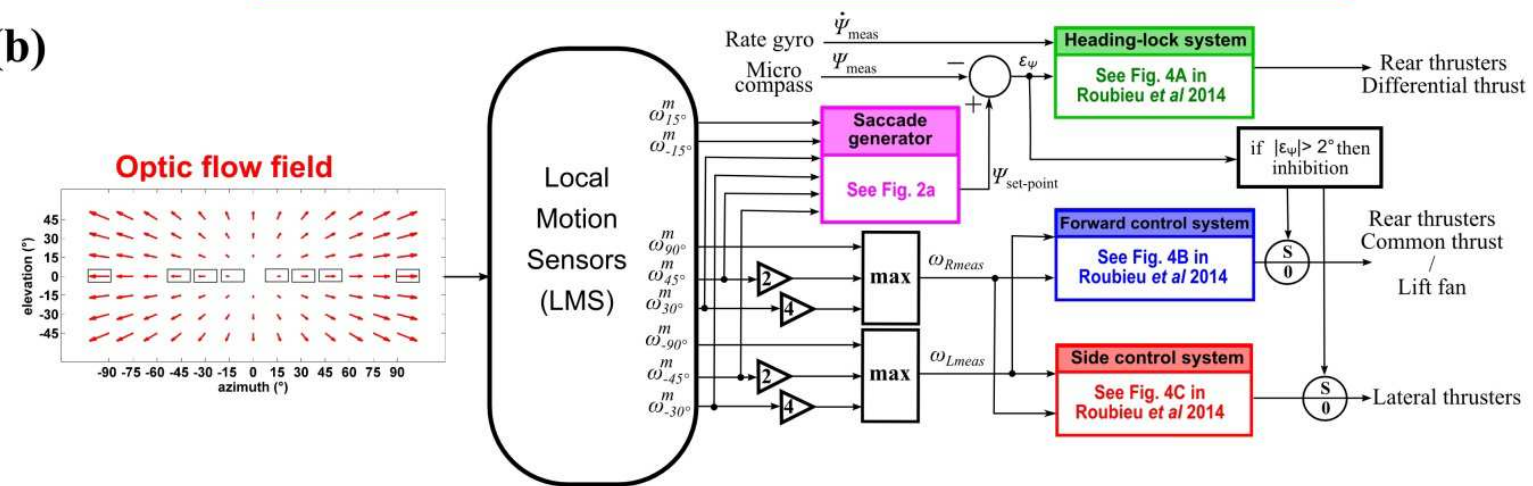

Fig. 2. (a) Functional description of the saccade generator (see §3.2). (b) Overview of the full control scheme described in terms of a Brooks' subsumption architecture. LMS stands for Local Motion Sensors, which spatially sample 8 local optic flow (OF) measurements. On the left: the inertial, magnetic and visual inputs (from 8 LMSs, 1 rate gyro and 1 micro compass) to the autopilot, and on the right: the robot's control output signals (to the rear and lateral thrusters and the lift fan). Under normal operating conditions, the robot's heading $\Psi$ is set at a constant value by the heading-lock system (Fig. 2a), and both the forward and side speeds are controlled by the $\mathrm{OF}$ (see §3.1). If an imminent collision with an obstacle is detected, the robot's incidence angle $i$ with respect to a frontal obstacle is then estimated from the OF measurements at $\omega_{ \pm 30^{\circ}}^{m}$ to compute and update the new robot's heading $\Psi_{\text {set-point }}$ (Fig. 2a). 


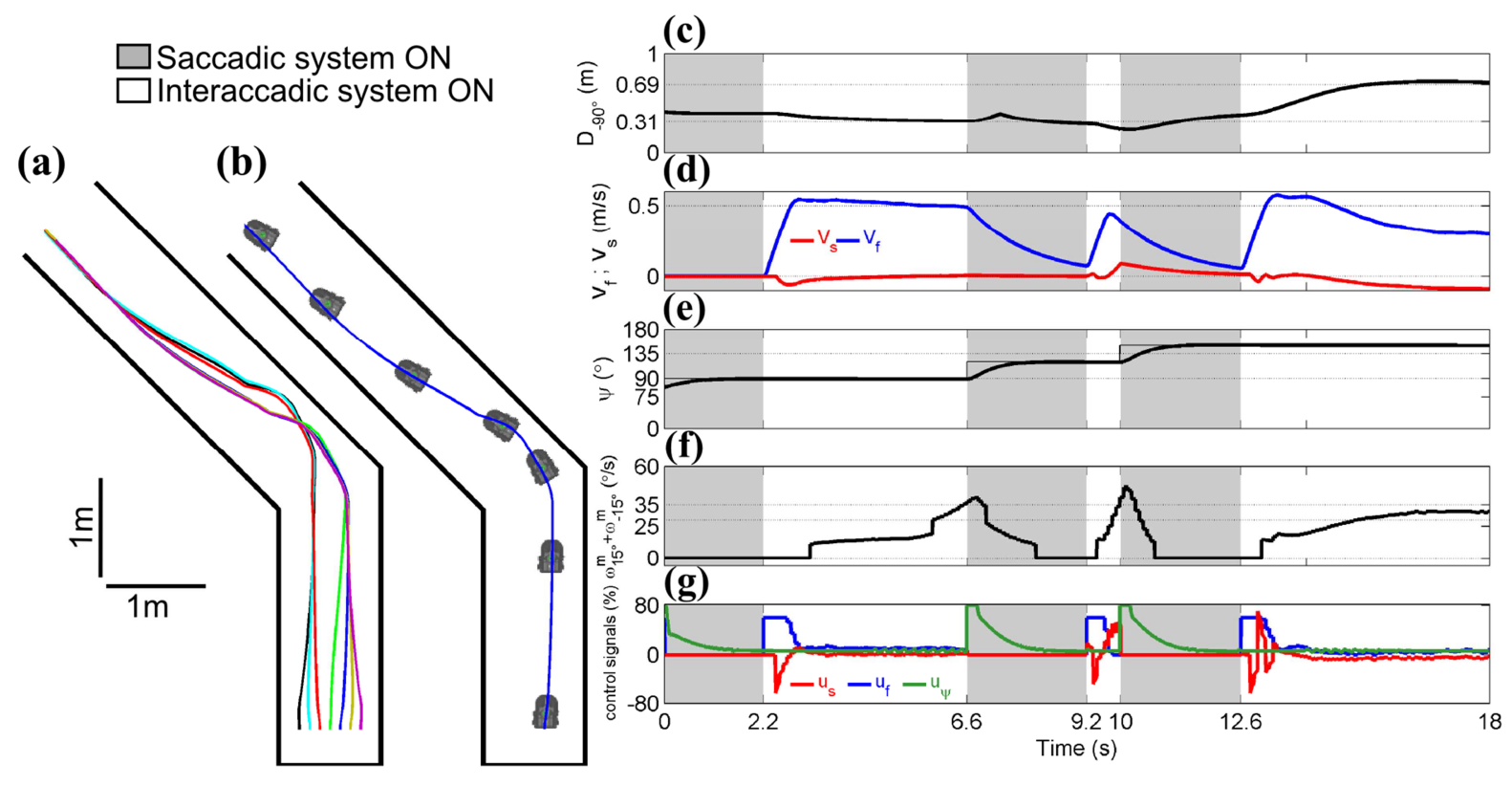

Fig. 3. (a) 7 simulated robot trajectories in a 1-meter wide corridor comprising a $45^{\circ}$-bend, starting at $x_{0}=$ (4.20m; 4.30m; 4.40m; 4.50m; 4.60m; 4.70m; 4.80m), $y_{0}=0.35 \mathrm{~m}$, and $\Psi_{0}=75^{\circ}$. (b) An individual robot trajectory. Marks on the trajectory indicate the robot's position and its orientation at every $2.8 \mathrm{~s}$. The robot started at a point located at $x_{0}=4.60 \mathrm{~m}, y_{0}=0.35 \mathrm{~m}$ in the corridor, oriented at $\Psi_{0}=75^{\circ}$ as in the blue trajectory presented in (a). (c) Lateral distance from the left wall $D_{-90^{\circ}}$ corresponding to the robot's trajectory shown in (b). (d) The forward speed $V_{f}$ profile (in blue) and the side speed $V_{s}$ profile (in red) corresponding to the robot's trajectory shown in (b). (e) The robot's orientation $\Psi$ (thick line) and the orientation set-point $\Psi_{\text {set-point }}($ thin line). (f) Sum of the optic flow measurements obtained at $\pm 15^{\circ}$ azimuthal angles corresponding to the robot's trajectory shown in (b). (g) The three control signals $u_{s}$ (the lateral control signal in red), $u_{f}$ (the forward control signal in blue), and $u_{\Psi}$ (the yaw control signal in green) corresponding to the robot's trajectory shown in (b). 

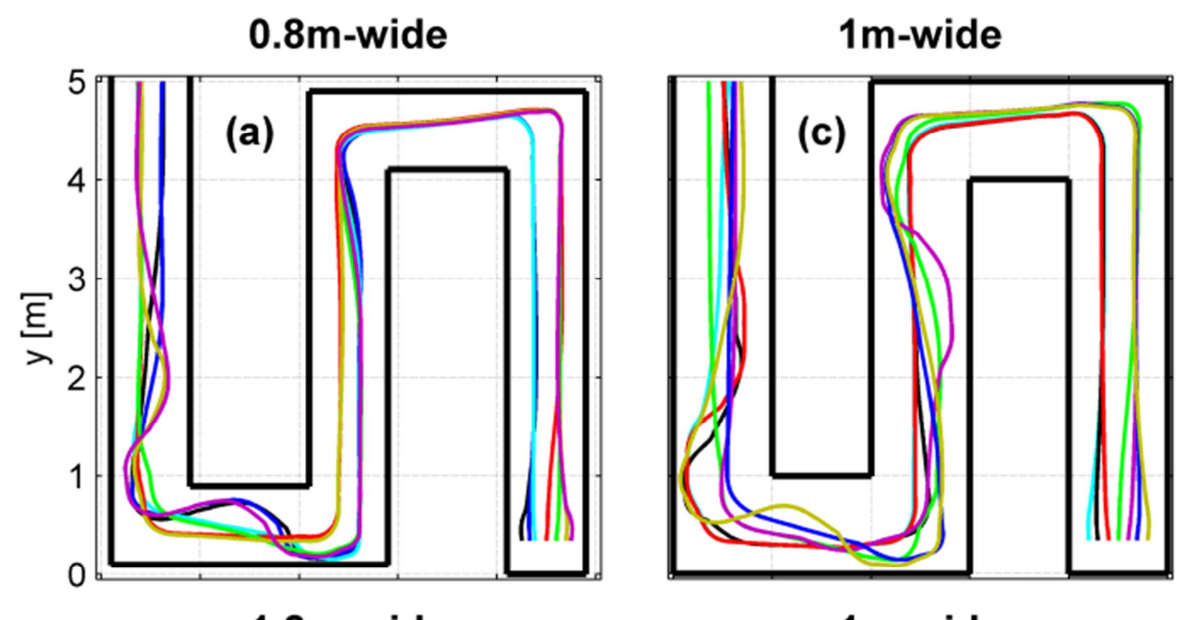

$1.2 \mathrm{~m}$-wide

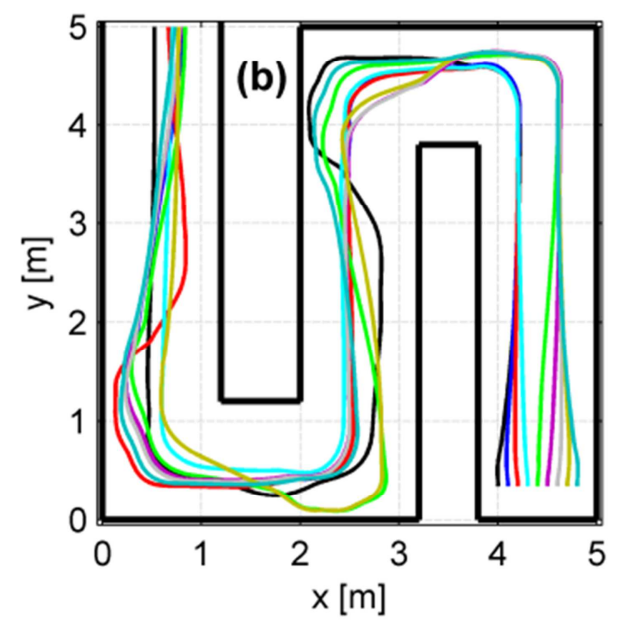

1m-wide

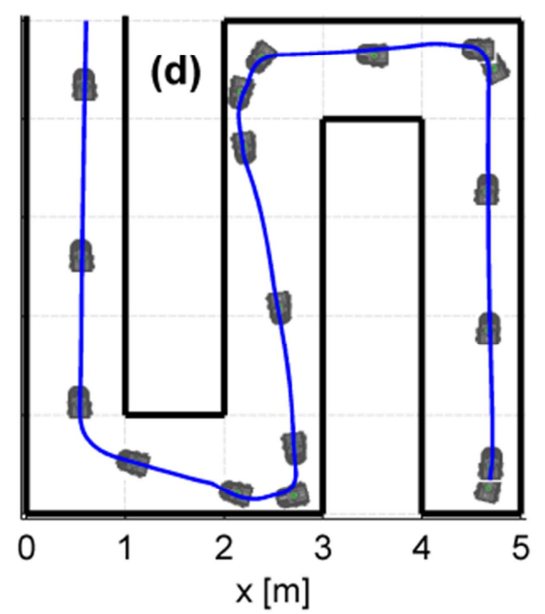

Fig. 4. Trajectories of the simulated robot in corridors of various widths comprising four L-junctions.

(a) 7 simulated robot's trajectories in a $0.80 \mathrm{~m}$-wide corridor, starting at $x_{0}=(4.25 \mathrm{~m} ; 4.35 \mathrm{~m} ; 4.40 \mathrm{~m} ; 4.50 \mathrm{~m}$; 4.60m; 4.70m; 4.75m), $y_{0}=0.35 \mathrm{~m}$, and $\Psi_{0}=75^{\circ}$.

(b) 9 simulated robot trajectories in a $1.20 \mathrm{~m}$-wide corridor, starting at $x_{0}=(4 \mathrm{~m} ; 4.10 \mathrm{~m} ; 4.20 \mathrm{~m} ; 4.30 \mathrm{~m} ; 4.40 \mathrm{~m}$; $4.50 \mathrm{~m} ; 4.60 \mathrm{~m} ; 4.70 \mathrm{~m} ; 4.80 \mathrm{~m}), y_{0}=0.35 \mathrm{~m}$, and $\Psi_{0}=75^{\circ}$.

(c) 7 simulated robot trajectories in a $1 \mathrm{~m}$-wide corridor, starting at $x_{0}=(4.20 \mathrm{~m} ; 4.30 \mathrm{~m} ; 4.40 \mathrm{~m} ; 4.50 \mathrm{~m} ; 4.60 \mathrm{~m}$; $4.70 \mathrm{~m} ; 4.80 \mathrm{~m}), y_{0}=0.35 \mathrm{~m}$, and $\Psi_{0}=75^{\circ}$.

(d) Individual robot trajectory (the blue trajectory presented in (c)). Marks on the trajectory indicate the robot's position and orientation at every $2.8 \mathrm{~s}$. The robot started at a point located at $x_{0}=4.80 \mathrm{~m}, y_{0}=0.35 \mathrm{~m}$ in the corridor and oriented at $\Psi_{0}=75^{\circ}$. 


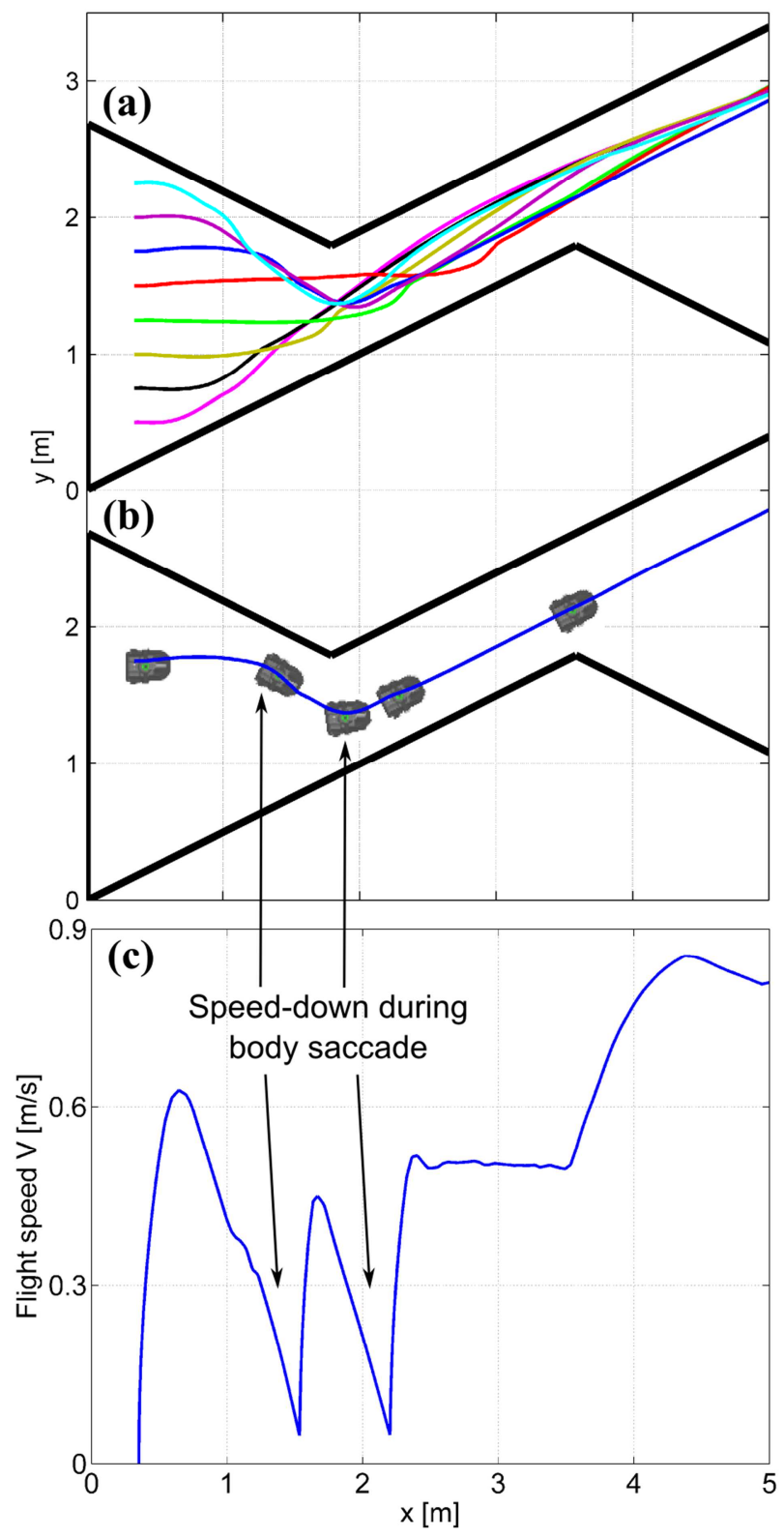

Fig. 5. (a) 8 simulated robot's trajectories in a tapered corridor including a straight junction located midway. The robot started at various initial ordinates corresponding to $y_{0}=(0.50 \mathrm{~m} ; 0.75 \mathrm{~m} ; 1 \mathrm{~m} ; 1.25 \mathrm{~m} ; 1.50 \mathrm{~m} ; 1.75 \mathrm{~m} ; 2 \mathrm{~m}$; $2.25 \mathrm{~m}$ ), an initial abscissa $x_{0}=0.35 \mathrm{~m}$, and an initial orientation $\Psi_{0}=15^{\circ}$. (b) Individual robot's trajectory (the blue trajectory presented in (a)). Marks on the trajectory indicate the robot's position and its orientation at every 2.8s. The robot started at a point located at $x_{0}=0.35 \mathrm{~m}, y_{0}=1.75 \mathrm{~m}$ in the corridor, oriented at $\Psi_{0}=15^{\circ}$. (c) Flight speed profile along the tapered corridor corresponding to the robot's trajectory shown in (b). 


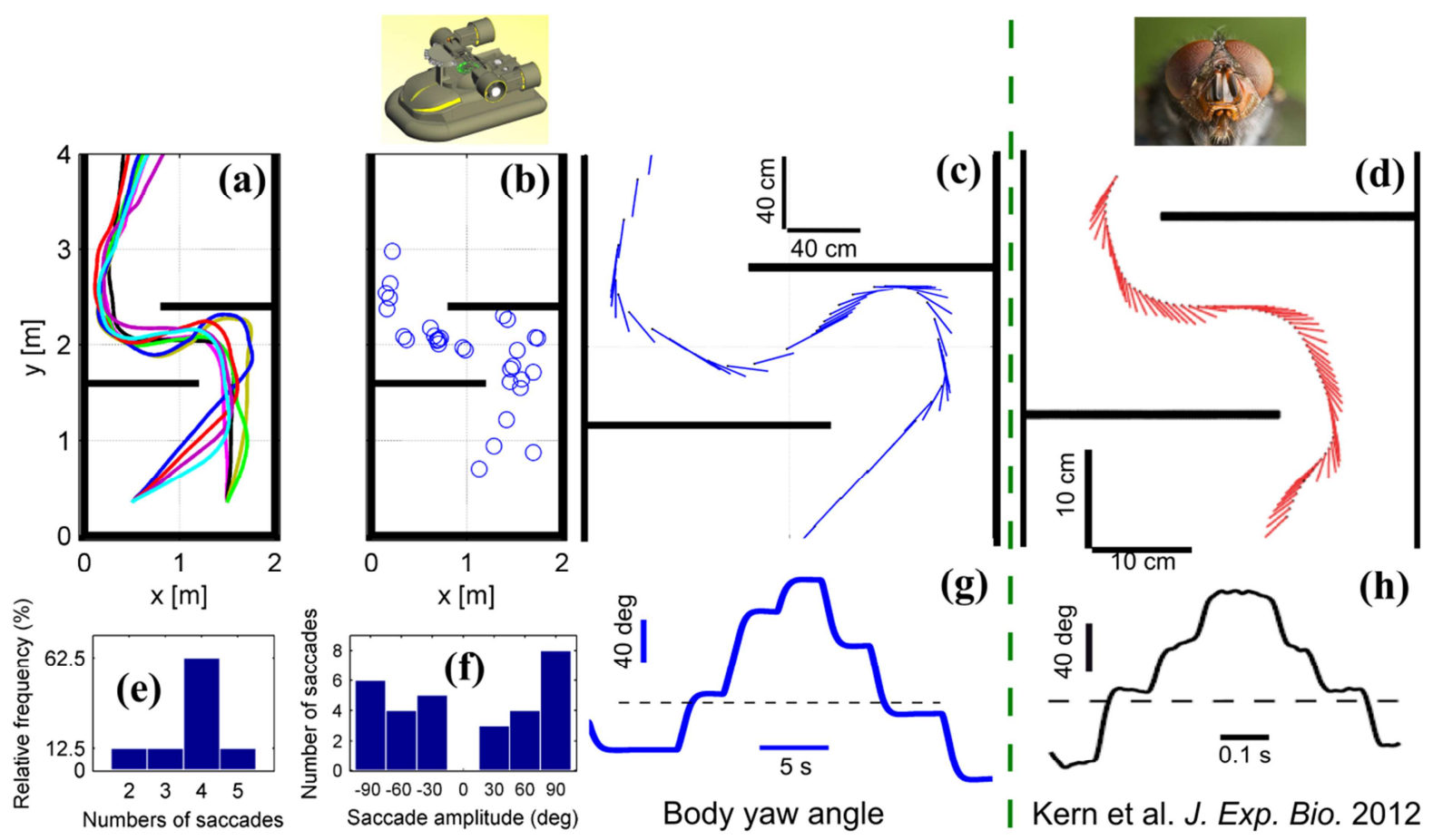

Fig. 6. Direct comparison between the behavior of the simulated robot and that of the blowfly in an S-shaped turn.

(a) 8 simulated robot's trajectories in an S-shaped turn, starting at an initial position $\left(x_{0}=0.5 \mathrm{~m} ; y_{0}=0.35 \mathrm{~m}\right)$ with various initial orientations $\Psi_{0}=\left(35^{\circ} ; 40^{\circ} ; 45^{\circ} ; 50^{\circ}\right)$, or an initial position $\left(x_{0}=1.5 \mathrm{~m} ; y_{0}=0.35 \mathrm{~m}\right)$ and various initial orientations $\Psi_{0}=\left(70^{\circ} ; 80^{\circ} ; 90^{\circ} ; 95^{\circ}\right)$.

(b) Points in the corridor at which the saccades occurred.

(c) Typical trajectory of the robot among the set of 8 trajectories presented in (a). The position of the robot (black circles) and its body orientation (blue lines) are plotted every 400ms.

(d) Typical trajectory of a blowfly in an S-shaped turn, adapted from data published by [27]. Position of the fly (black circles) and its long body axis (red lines) are plotted every 10ms. Inset: head of a Calliphora vomitoria (Picture: J. J. Harrison, Wikimedia commons).

(e) Relative frequency of saccades per trajectory when crossing the S-shaped turn $(n=30)$.

(f) Body saccades were classified depending on their amplitude: $-90^{\circ}$, between $-89^{\circ}$ and $-60^{\circ}$, between $-59^{\circ}$ and $30^{\circ}$, then between $30^{\circ}$ and $59^{\circ}$, between $60^{\circ}$ and $89^{\circ}$, and $90^{\circ}$.

(g) Corresponding time courses of the body yaw angle during the trajectory shown in (c). The horizontal dashed line gives the corridor's Y-axis $\left(\Psi=90^{\circ}\right)$.

(h) Corresponding time courses of body orientation during the trajectory shown in (d). Horizontal dashed line indicates the tunnel $\mathrm{Y}$-axis $\left(\Psi=90^{\circ}\right)$. Data adapted from [27]. 